\title{
The Synaptotagmin-1 C2B Domain Is a Key Regulator in the Stabilization of the Fusion Pore
}

\author{
Marcelo Caparotta, Claudia N. Tomes, Luis S. Mayorga, and Diego Masone* \\ Cite This: https://dx.doi.org/10.1021/acs.jctc.0c00734 \\ Read Online
}

ACCESS I

Lلll Metrics \& More

Article Recommendations

Supporting Information

ABSTRACT: Fusion pores serve as an effective mechanism to connect intracellular organelles and release vesicle contents during exocytosis. A complex lipid rearrangement takes place as membranes approximate, bend, fuse, and establish a traversing water channel to define the fusion pore, linking initially isolated chambers. Thermodynamically, the process is unfavorable and thought to be mediated by specialized proteins. In this work, we have developed a reaction coordinate to induce fusion pores from initially flat and parallel lipid bilayers and we have used it to describe the

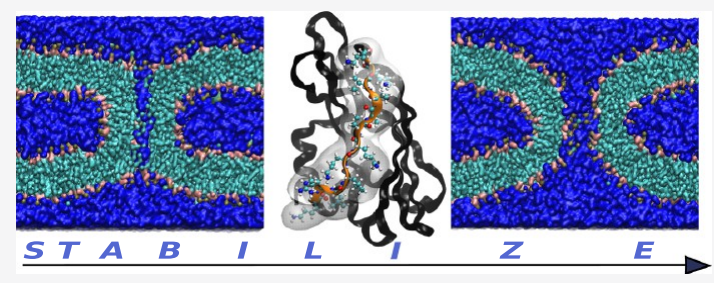
effects of the synaptotagmin-1 C2B domain during the process. We have obtained free-energy profiles of the whole lipid reorganization in biologically realistic membranes, going from planar and parallel bilayers through stalk hemifusion to water channel formation. Our results point to a lysine-rich polybasic region on synaptotagmin-1 C2B as the key to lipid reorganization control through the formation of phosphatidylinositol bisphosphate clusters that stabilize the fusion pore.

\section{INTRODUCTION}

Membrane fusion and poration have been largely studied by computational $^{1-10}$ and experimental means. ${ }^{1{ }^{13}}$ Among the most accepted mechanisms for the complex reorganization of the lipid molecules while shaping a fusion pore are those of stalk formation, ${ }^{14}$ inverted micelles, ${ }^{15}$ stalk bending, ${ }^{16}$ and elongation. $^{17}$

Computational simulations are particularly suitable to study transient and metastable states (such as hemifusion) as intermediate steps prior to a permanent final configuration. Although many studies rely on different types of molecular dynamics simulations, ${ }^{1-9}$ other techniques have also been used to describe these phenomena, i.e., continuum models, ${ }^{18,19}$ selfconsistent field theory, ${ }^{20}$ dissipative particle dynamics, ${ }^{21}$ particle simulations, and string methods. ${ }^{2,23}$

Synaptotagmin-1 (Syt1) is a low-affinity $\mathrm{Ca}^{2+}$ sensor that binds and penetrates the biomembrane to trigger vesicle fusion and neurotransmitter release. ${ }^{24-26}$ It contains two C2 domains (C2A and C2B) with similar structures, both with $\mathrm{Ca}^{2+}$ binding loops. These loops control penetration into biomembranes containing phosphatidylserines. ${ }^{27-29}$ Moreover, Synaptotagmin-1 $\mathrm{C} 2 \mathrm{~B}$ is known to bind phosphatidylinositol-4,5bisphosphate $\left(\mathrm{PIP}_{2}\right)$ in a $\mathrm{Ca}^{2+}$-independent manner. ${ }^{30-33}$ Consequently, the synaptotagmin-1 $\mathrm{C} 2 \mathrm{~B}$ domain is known as a phospholipid-binding machine ${ }^{34}$ and has been related to membrane deformation, synaptic vesicle fusion, ${ }^{35}$ fusion pore opening, ${ }^{36}$ stabilization, ${ }^{37}$ and expansion. ${ }^{38}$

The relatively large size of $\mathrm{PIP}_{2}$ lipids and their erect structure allow them to protrude further into the aqueous phase than many other phospholipids. ${ }^{39} \mathrm{PIP}_{2}$ has been involved in the regulation of a variety of cellular processes, such as synaptic vesicle trafficking, ${ }^{40}$ exocytosis, endocytosis, and enzyme activation. ${ }^{39,41}$ Several studies have described $\mathrm{PIP}_{2}$ specific binding to $\mathrm{C} 2$ domains, ${ }^{30,42-44}$ and it is well established that $\mathrm{PIP}_{2}$ serves to anchor proteins to the plasma membrane. ${ }^{39}$ Interestingly, $\mathrm{PIP}_{2}$ clusters have been reported to function as molecular beacons during vesicle recruitment. ${ }^{45,46}$

In particular, polybasic patches (similar to KRLKKKKTTIKK, positions $321-332$ in the rat synaptotagmin-1 C2B domain, PDB ID: 1k5w) have been suggested to directly bind $\mathrm{PIP}_{2}$ lipids. ${ }^{45,47,48}$ Figure 1 shows the synaptotagmin-1 C2B domain in its atomistic (a) and coarse-grained (c) representations, together with a detailed view of the polybasic region KRLKKKKTTIKK (positions 321-332) (b). Additional evidence show that (i) sytnaptotagmin-1 C2 domains modulate the rate expansion of the fusion pore $^{38}$ with polybasic patches playing a key role, ${ }^{48}$ (ii) $\mathrm{PIP}_{2}$ increases the speed of response of synaptotagmin by steering its membrane-penetration activity toward the plasma membrane, ${ }^{30}$ and (iii) intense protein-lipid electrostatic interactions facilitate mutual local enrichment, inducing protein and $\mathrm{PIP}_{2}$ microdomains at the fusion sites. ${ }^{46}$ Altogether, these findings point to the importance of $\mathrm{PIP}_{2}$ strong anionic interactions with polybasic patches of synaptotagmin $\mathrm{C} 2$ domains in processes involving membrane bending ${ }^{45,49-51}$ and fusion. ${ }^{50,52-54}$

Received: July 14, 2020 

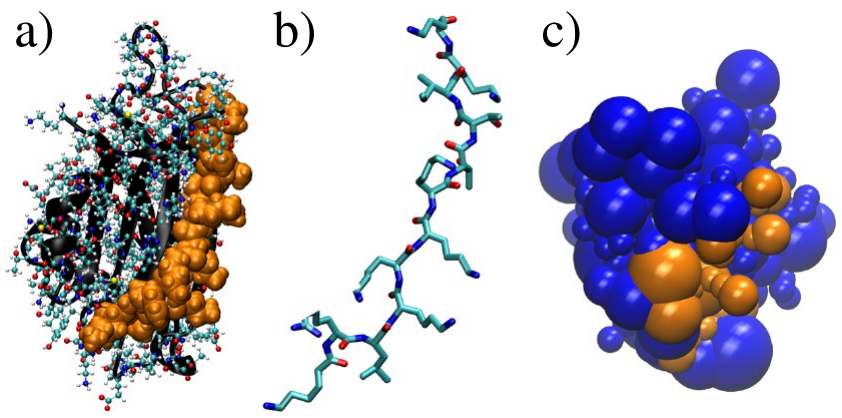

Figure 1. Synaptotagmin-1 C2B domain representations. (a) Atomistic (PDB ID: $1 \mathrm{k} 5 \mathrm{w}$ ) with the polybasic region highlighted in orange van der Waals surfaces. Ribbons for the whole domain are black, and the rest of the amino acids follow the Corey-PaulingKoltun (CPK) coloring convention for distinguishing atoms. (b) Detailed stick representation of the polybasic region KRLKKKKTTIKK (positions 321-332). (c) MARTINI coarsegrained beads with the polybasic region highlighted in orange.

Importantly, Park and $\mathrm{Ryu}^{53}$ proposed that the polybasic region in the $\mathrm{C} 2 \mathrm{~B}$ domain selectively interacts with $\mathrm{PIP}_{2}$ lipids, inducing $\mathrm{C} 2 \mathrm{~B}$-domain binding to the plasma membrane and triggering vesicle fusion. $\mathrm{Wu}$ et al. ${ }^{13}$ recently suggested that stnaptotagmin-1-mediated membrane bending facilitates the opening of an initial fusion pore by helping to bring the two membranes together, reducing the repulsive hydration forces, and exposing their hydrophobic interiors to initiate lipid exchange. However, the molecular mechanisms for this regulation remain less clear. ${ }^{13}$

In this work, we have used computational simulations to mimic biological intracellular organelles as they approximate, fuse, and connect themselves through a fusion pore. Introducing for the first time a reaction coordinate that induces the formation of a fusion pore, we have obtained freeenergy profiles for fusion pore nucleation (the first step in the formation of a new configuration). To do so, we have modified the original implementation of reaction coordinate $\xi$ by Hub and Awasthi, ${ }^{8}$ which induces a hydrophilic pore in a single lipid bilayer (source code generously provided by Prof. J. Hub through personal communication). Our modified version of $\xi$, defined $\xi^{\prime}$ from now on, induces two initially planar and parallel membranes to bend, fuse, and form a thin hydrophilic channel traversing them to establish the fusion pore.

We have used our computational methodology to evaluate the effects of synaptotagmin-1 $\mathrm{C} 2 \mathrm{~B}$ in the process, showing that the $\mathrm{C} 2 \mathrm{~B}$ domain does not interfere with membrane fusion nor initial water channel formation. However, as the fusion pore expands, the $\mathrm{C} 2 \mathrm{~B}$ domain effects become noticeable through strong interactions between its polybasic region KRLKKKKTTIKK (positions 321-332) and phosphatidylinositol bisphosphate lipids. Our results show that, by inducing the formation of phosphatidylinositol bisphosphate lipid clusters, the $\mathrm{C} 2 \mathrm{~B}$ domain stabilizes the fusion pore.

\section{RESULTS AND DISCUSSION}

In computational biology, enhanced sampling has been widely used to study configurations of interest that are in general difficult to reach by standard dynamics. ${ }^{5-57}$ Popular and stateof-the-art methods to estimate free energies (i.e., umbrella sampling ${ }^{58,59}$ and well-tempered metadynamics ${ }^{60-62}$ ) are, unfortunately, highly dependent on the correct formulation of the reaction coordinate that better projects the multidimen- sional free-energy surface. ${ }^{63}$ Therefore, the development of new reaction coordinates describing collective phenomena is of major importance to bias molecular dynamics simulations and calculate the associated free-energy landscapes. Thus, the accurate description of a rare event (such as the formation of a fusion pore) by a closed-form mathematical expression is very much desirable.

Accordingly, Müller and co-workers ${ }^{64}$ reviewed several methods for calculating free energies of collective processes in membranes that involve the cooperative reorganization of many molecules. Hub and Awasthi ${ }^{9}$ reviewed and ran tests for convergence, hysteresis, and finite-size effects for three different well-known reaction coordinates commonly used for pore formation in a single bilayer. Mirjalili and $\mathrm{Feig}^{7}$ presented a new reaction coordinate to bias molecular dynamics simulations in density-driven processes to induce a hydrophilic single-membrane pore. Finally, Hub and Awasthi ${ }^{8}$ developed a novel reaction coordinate to induce a continuous polar defect in a lipid bilayer. Importantly, this last reaction coordinate exhibits virtually no hysteresis and is able to restrain the system close to the transition state.

Concentric Cylinders' Reaction Coordinate for Fusion Pore Formation. Inspired by the original work by Hub and Awasthi, ${ }^{8}$ we defined two cylinders normal to the planes of the bilayers (see Figure 2) and performed umbrella sampling

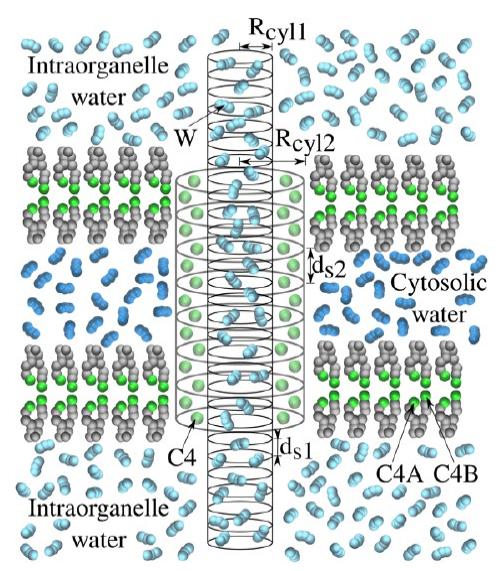

Figure 2. Concentric cylinders' reaction coordinate mechanism. Cylinder 2 fills with C4 lipid beads, forcing membranes to fuse while the water channel forms inside cylinder 1. Lipids (gray) accommodate themselves (tilting and splaying) to fulfill the reaction coordinate imposition as the fusion pore forms. The green beads are the $\mathrm{C} 4$ at the end of the lipid tails, cytosolic water molecules are blue, and intraorganelle water molecules are cyan.

simultaneously using two reaction coordinates: $\xi_{1}^{\prime}$ and $\xi_{2}^{\prime}$. Accordingly, two independent biasing potentials with force constants $k_{1}$ and $k_{2}$ were applied to two different groups of beads: $\mathrm{W}$ beads in intraorganelle waters and $\mathrm{C} 4$ tail beads in lipid molecules. The first reaction coordinate $\left(\xi_{1}^{\prime}\right)$ accounts for the fraction of cylinder slices occupied by intraorganelle water molecules (in resemblance to the internal content of two organelles about to fuse), defined as all $\mathrm{W}$ beads above and below the initially flat and parallel membranes (cyan). The second reaction coordinate $\left(\xi_{2}^{\prime}\right)$ is defined as the fraction of slices occupied by $\mathrm{C} 4 \mathrm{~A}$ or $\mathrm{C} 4 \mathrm{~B}$ beads in lipid tails (green), which is used here to fuse the membranes by tilting lipid molecules and to radially displace cytosolic waters (blue). This pulling strategy tilts the nearest lipids toward the fusion patch, 
inducing a hydrophobic core and lipid splaying (see molecular dynamics insets in Figure 5a). Subsequently, the systems go into stalk hemifusion as already observed in fusion pores driven by the neuronal SNARE complex ${ }^{6}$ and in barrier crossing during liposome fusion. ${ }^{65}$

Both $\xi_{1}^{\prime}$ and $\xi_{2}^{\prime}$ are dimensionless reaction coordinates mathematically identical to $\xi$, as defined by Hub and Awasthi, ${ }^{8}$ with geometrical parameters adjusted to sequentially induce membrane fusion and water channel formation (for details, see "Reaction Coordinate Parameters: Equalization and Dependence"). Only for a straightforward comparison between different free-energy profiles projected in $1 \mathrm{D}$ and not for biasing simulations, we propose here $\xi^{\prime}$ as the mean value between $\xi_{1}^{\prime}$ and $\xi_{2}^{\prime}$, (see eq 1 ).

$$
\xi^{\prime}=\frac{1}{2}\left(\xi_{1}^{\prime}+\xi_{2}^{\prime}\right)
$$

The definition of $\xi^{\prime}$ in eq 1 fits the approach used by GROMACS in gmx wham ${ }^{66}$ to conveniently calculate $1 D$ freeenergy profiles when multiple restraints are present (for more details, see GROMACS 2018.3 documentation at http:// manual.gromacs.org/). Alternatively, free-energy surfaces calculated with WHAM-2D ${ }^{67}$ for the equivalent visualization of the free energy as a function of both variables $\xi_{1}^{\prime}$ and $\xi_{2}^{\prime}$ are provided in Figure S6 in the Supporting Information. Like the original reaction coordinate, $\xi^{\prime}$ is also continuous and differentiable and shows no significant hysteresis (see the "Hysteresis-Free Sampling" section).

Following an event-oriented classification of the different stages of the fusion pore, as described in the literature, ${ }^{6,13,23,68-70}$ the process to induce a fusion pore starts with two flat and parallel separated bilayers $\left(\xi^{\prime} \approx 0.2\right)$. The first stage is membrane bending $\left(0.2<\xi^{\prime}<0.7\right)$ with apposed local deformations of the bilayers where the lipids in the upcoming fusion patch begin to tilt ( $\xi^{\prime}$ pulling is applied to tail $\mathrm{C} 4$ beads $)$. By the end of this stage $\left(\xi^{\prime} \approx 0.6\right)$, splayed lipids can be observed transiently connecting the head-group regions and leading to hemifusion (see molecular dynamics insets in Figure 5a). The second stage is membrane fusion to form the stalk with membrane mutual exposure of hydrophobic interiors $\left(0.7<\xi^{\prime}<0.9\right)$. The third stage is fusion pore nucleation: as understood in thermodynamics (with particular biological interest), nucleation is the first step in the formation of a new structure, corresponding here to the establishment of the first water channel traversing the fusion patch $\left(0.9<\xi^{\prime}<1\right)$. We have set the water channel radius to $R_{\text {cyll }}=0.8 \mathrm{~nm}$ in agreement with the nucleation of a hydrophilic pore in a single lipid bilayer. $^{8}$

Finally, a fourth stage is the widening of the water channel to expand the fusion pore by increasing both cylinder radii $\left(R_{\text {cyll }}\right.$ and $R_{\text {cyl } 2}$; see Figure 2). Biasing different double-cylinder geometries, we have found target values of $R_{\text {cyll }}=2.5 \mathrm{~nm}$ and $R_{\text {cyl2 }}=5 \mathrm{~nm}$ to fluently drive the fusion pore beyond any possible hidden energetic barrier that may exist between nucleation and expansion. Interestingly, among these stages, previous studies have identified the pore expansion stage to be the energetically costliest one. ${ }^{13,71-73}$

Reaction Coordinate Parameters: Equalization and Dependence. As it occurs biologically, the content of the organelles does not make contact with the exterior of the cells, making these definitions of water molecule groups a direct analogy of what happens in vivo (in Figure 2, water molecule groups in cyan and blue are mutually exclusive). The cylinder geometries are adjustable by the user, i.e., the number of cylinder slices $\left(N_{\mathrm{s}}\right)$, their thickness $\left(d_{s}\right)$, their radius $\left(R_{\text {cyl }}\right)$, and their occupation factor $(\zeta)$, defined according to the original nomenclature. ${ }^{8}$ Importantly, $\xi^{\prime}$ is defined as an exclusive function of membrane and water molecules, capturing only indirectly any effects on the bilayers due to, for example, an interacting protein.

In principle, $\xi_{1}^{\prime}$ and $\xi_{2}^{\prime}$ individually share the same mathematical formulation as $\xi$ by Hub and Awasthi. ${ }^{8}$ However, the simultaneous application of $\xi_{1}^{\prime}$ and $\xi_{2}^{\prime}$ to different groups of beads in a system containing two lipid bilayers presents a different scenario. We have modified the pulling code developed for $\xi$ to use two sliced cylinders at the same time in order to independently bias different groups of beads, conveniently chosen to subsequently induce membrane fusion and water channel formation. Analogous to what was emphasized by Hub and Awasthi for their own reaction coordinate for pores in single lipid bilayers, ${ }^{8}$ using different sets of parameters, $R_{\text {cyl } 1}, R_{\text {cyl } 2}, d_{s 1}, d_{s 2}, \zeta_{1}$, and $\zeta_{2}$ define different reaction coordinates each one in its own phase space.

The process of biasing $\xi_{1}^{\prime}$ and $\xi_{2}^{\prime}$ to induce the fusion pore starts by fusing the bilayers, which can only happen if the outer cylinder pulling C4 lipid beads is narrow enough to merge the initially opposed bilayers in a single contact region but still wide enough to leave sufficient space for the upcoming water channel. Large values of $R_{\text {cyl2 }}$ (i.e, $R_{\text {cyl2 }}>2 \mathrm{~nm}$ ) do not produce any membrane fusion as the cylinder easily fulfills the reaction coordinate condition with local membrane deformations that do not connect. On the other hand, low values of $R_{\text {cyl2 }}$ (i.e., $R_{\text {cyl } 2}<1.5 \mathrm{~nm}$ ) do not allow for the water channel to form, leaving the system (in the best of cases) in the stalk hemifusion stage. On top of this, the inner cylinder controlling the water channel needs to be tall enough to reach the intraorganelle waters (above the top bilayer and below the bottom one). At the same time, it must be narrower as possible to avoid hysteresis. Then, $R_{\text {cyll }}$ was set to the minimum value $\left(R_{\text {cyll }}=\right.$ $0.8 \mathrm{~nm}$ ) that allows for the thinnest water channel to connect, inducing fusion pore nucleation. This same value $(0.8 \mathrm{~nm})$ was suggested by $\mathrm{Hub}$ and $\mathrm{Awasthi}^{8}$ to be adequate to nucleate a hydrophilic pore in a single lipid bilayer.

Parameters $d_{s 1}$ and $d_{s 2}$ determining the thickness of the slices were set to 0.4 and $0.2 \mathrm{~nm}$, respectively, to keep the amount of slices constant in both cylinders $\left(N_{s}=30\right)$. The occupation factor $\zeta_{1}=0.75$ was kept as in Hub and Awasthi. ${ }^{8}$ However, $\zeta_{2}$ was set to a much lower value $\left(\zeta_{2}=0.35\right)$ to force the outer cylinder to gather more $\mathrm{C} 4$ lipid beads to fulfill the reaction coordinate imposition, hence favoring membrane fusion. Everything considered unsuitable sets of parameters for $\xi_{1}^{\prime}$ and $\xi_{2}^{\prime}$ either do not produce a unique membrane fusion patch or do not induce a thin water channel where the membranes fuse, which, in either case, do not lead to a fusion pore.

As originally defined by Hub and Awasthi, cylinder slice occupation rapidly saturates after one bead is inside the slice, meaning that the reaction coordinate is strongly increased by the addition of the first bead but only marginally increased by the addition of a second or a third bead to the same slice. ${ }^{8}$ Taking advantage of this property, when the pore expands (by increasing both $R_{\text {cyll }}$ and $R_{\text {cyll }}$ ), the water channel remains always connected as its widening happens in the saturation zone of the reaction coordinate. This feature has become useful to describe the effects of the synaptotagmin-1 C2B domain on fusion pore stabilization. 
As the scope of this work is to study the effects of the synaptotagmin-1 C2B domain on the dynamics of the fusion pore, we have chosen an amount of cytosolic water molecules (confined between the bilayers) that equilibrates the intermembrane separation distance at $\sim 3.5 \mathrm{~nm}$ in order to fit one $\mathrm{C} 2 \mathrm{~B}$ domain. Higher intermembrane separation distances resulted to be inconvenient for the synaptotagmin$1 \mathrm{C} 2 \mathrm{~B}$ domain to interact with both bilayers simultaneously and thus not suitable for this study. Furthermore, for the fusion pore to still form, larger intermembrane separations (more cytosolic water) would require taller cylinders (increasing $N_{\mathrm{s}}=$ 30 and/or $d_{s}$ ) but not necessarily wider ones, significantly altering their aspect ratios (see Figure S10 in the Supporting Information for molecular dynamics snapshots of a system with $\sim 35 \%$ more cytosolic water molecules). This situation would again ask for a different set of parameters that would define a different reaction coordinate in a different phase space. Difficulties in parameter tuning are inherent to the slicedcylinder mechanism of the reaction coordinate and were also highlighted by $\mathrm{Hub}$ and Awasthi for their own reaction coordinate. For a detailed description of hydration effects on membrane fusion due to varying intermembrane distances, see the work of Smirnova et al. ${ }^{23}$

As a corollary, we have used the same concentric cylinders in Figure 2 to expand the fusion pore once it has been formed. Starting from a configuration with $\xi^{\prime} \approx 1$ where both cylinders are completely full with their respective beads and keeping constant the biasing potential that controls cylinder 1 (water channel), we were able to expand the fusion pore by simply setting larger cylinder radii $\left(R_{\text {cyll }}\right.$ and $\left.R_{\text {cyl2 }}\right)$. Under this scheme, cylinder 1 is always completely occupied, keeping the water channel always connected, while biasing potential 2 displaces more C4 lipid beads radially away from the water channel, making the fusion pore expand.

In all fusion pore nucleation simulations performed in this study, we used two cylinders containing 30 slices $\left(N_{s}=30\right)$ each. Cylinder sizes were set differently as cylinder 1 is contained inside cylinder 2 and they are concentric (a water channel forms where membranes fuse). Namely, cylinder 1 accounting for intraorganelle $\mathrm{W}$ beads is thinner and taller $\left(R_{\text {cyll }}=0.8 \mathrm{~nm}, d_{s 1}=0.4 \mathrm{~nm}, \zeta_{1}=0.75\right)$ and is inside cylinder 2 accounting for C4 lipid beads $\left(R_{\text {cyl2 }}=1.8 \mathrm{~nm}, d_{s 2}=0.2 \mathrm{~nm}, \zeta_{2}\right.$ $=0.35)$; see Figure 2. In simulations involving fusion pore expansion, we used again two cylinders containing 30 slices $\left(N_{\mathrm{s}}\right.$ $=30$ ) each with parameters $R_{\text {cyll }}=2.5 \mathrm{~nm}, d_{s 1}=0.4 \mathrm{~nm}, \zeta_{1}=$ 0.65 and $R_{\text {cyl2 }}=5 \mathrm{~nm}, d_{s 2}=0.2 \mathrm{~nm}, \zeta_{2}=0.35$. The groups' definitions were kept identical to those of the nucleation of the fusion pore.

Energetics of the Fusion Pore in DPPC Bilayers. To validate our computational methodology, we obtained the freeenergy profile to induce a fusion pore between two initially flat and parallel pure dipalmitoylphosphatidylcholine (DPPC) model lipid bilayers.

As extensively reported in the literature, the thermodynamics of pore nucleation in a single lipid bilayer highly depends on the lipid composition. ${ }^{74,75}$ Using atomistic molecular dynamics, Wohlert and co-workers reported $\sim 300$ $\mathrm{kJ} / \mathrm{mol}$ as the free-energy cost to form a transmembrane pore of an $\sim 1.5 \mathrm{~nm}$ radius in a pure DPPC bilayer. ${ }^{1}$ Also, for atomistic DPPC bilayers, Mirjalili and $\mathrm{Feig}^{7}$ reported $\sim 93 \mathrm{~kJ} /$ mol, while Bennett et al. ${ }^{5}$ reported $\sim 78 \mathrm{~kJ} / \mathrm{mol}$ for DPPC, $\sim 17$ $\mathrm{kJ} / \mathrm{mol}$ for DLPC, and $\sim 45 \mathrm{~kJ} / \mathrm{mol}$ for DMPC. Hub and Awasthi ${ }^{8}$ reported $\sim 50 \mathrm{~kJ} / \mathrm{mol}$ for DMPC bilayers using their original reaction coordinate $\xi$ whose implementation we have adapted here to the fusion pore problem. In another study, ${ }^{9}$ Hub and Awasthi reviewed and tested different reaction coordinates and system sizes for atomistic DMPC bilayers finding values between $\sim 50$ and $\sim 250 \mathrm{~kJ} / \mathrm{mol}$. Using MARTINI coarse-grained molecular dynamics for DPPC, $\mathrm{Hu}$ et al. reported $\sim 300 \mathrm{~kJ} / \mathrm{mol}^{76}{ }^{76}$ For DOPC using Slipids, ${ }^{77}$ Batishchev and co-workers reported $\sim 100 \mathrm{~kJ} / \mathrm{mol}$.

Using two bilayers to induce a fusion pore, Kawamoto and Shinoda ${ }^{68,78}$ used a guided fusion pore through the stalk mechanism using coarse-grained molecular dynamics and continuum model simulations with their own SDK forcefield $^{79}$ for different membranes. They report $\sim 100 k_{\mathrm{B}} T$ for DOPC:DOPE, $\sim 120 k_{\mathrm{B}} T$ for DMPC:DOPE, $\sim 200 k_{\mathrm{B}} T$ for DMPC:DOPC, and $\sim 250 k_{\mathrm{B}} T$ for pure DMPC.

In agreement with these free-energy ranges, we report $\sim 160$ $k_{\mathrm{B}} T(\sim 400 \mathrm{~kJ} / \mathrm{mol})$ as the total cost to nucleate a fusion pore between two initially flat and parallel pure DPPC bilayers using MARTINI and GROMACS. In Figure 3, the free-energy

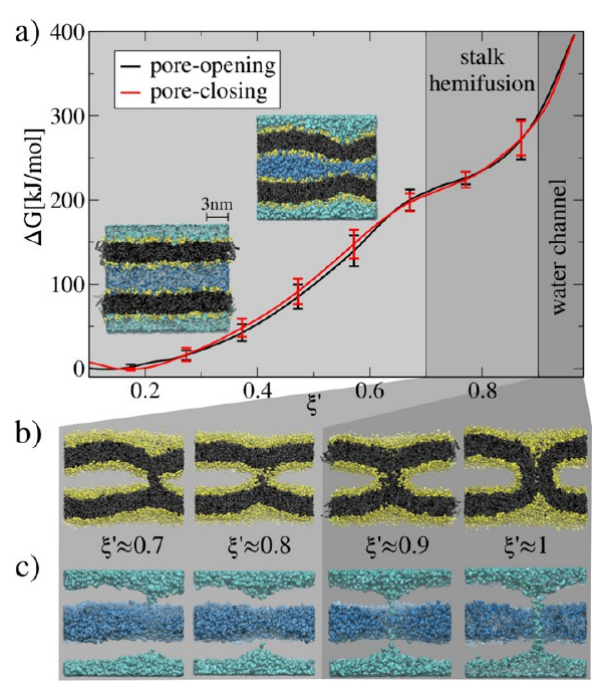

Figure 3. Fusion pore nucleation between two pure DPPC lipid bilayers. (a) Free-energy profiles for spanning reaction coordinate $\xi^{\prime}$ from $\sim 0.2$ (two planar and parallel bilayers) to $\sim 1$ (nucleated fusion pore) and backward. No significant hysteresis is observed between profiles corresponding to pore-opening (black) and pore-closing (red) slow growth paths. Inset snapshots illustrate the fusion pore evolution. Lipid molecules are black, cytosolic water is blue, and intraorganelle water is cyan. PO4, NC3, and GL beads are highlighted in yellow. Error bars are standard errors calculated by individually splitting the profiles in independent blocks. (b) Side view of lipid molecules only, showing lipid stalk hemifusion and water-channel formation events. (c) Side view of water molecules only. See the Supporting Information for PMF convergence and umbrella sampling technical details.

profile is shown together with molecular dynamics snapshots from umbrella sampling, depicting the transient configurations during fusion pore nucleation (PO4, NC3, and GL beads are highlighted in yellow, the rest of the lipid beads are black, blue was used for cytosolic water and cyan for intraorganelle water).

Relatively little bilayer deformation is noticeable for $\xi^{\prime}<0.6$ where the free energy profile exhibits a quadratic behavior ${ }^{1,80}$ accounting for membrane deformation and rearrangement of the lipids. For $0.6<\xi^{\prime}<0.7$, a change of the slope and regime takes place when bilayer bending rapidly increases and the freeenergy curve becomes 12th order-like for the rest of the 
a)

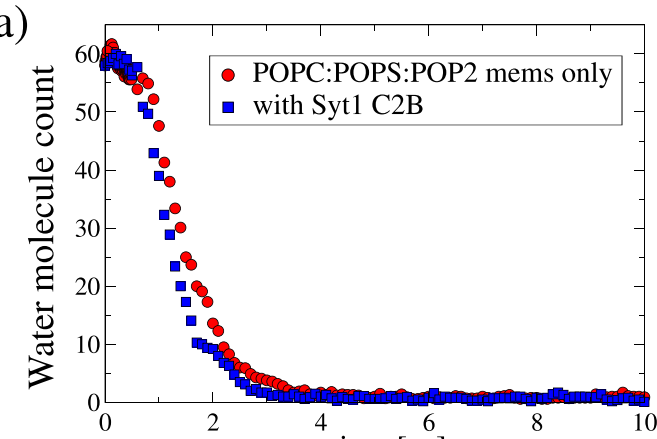

b)
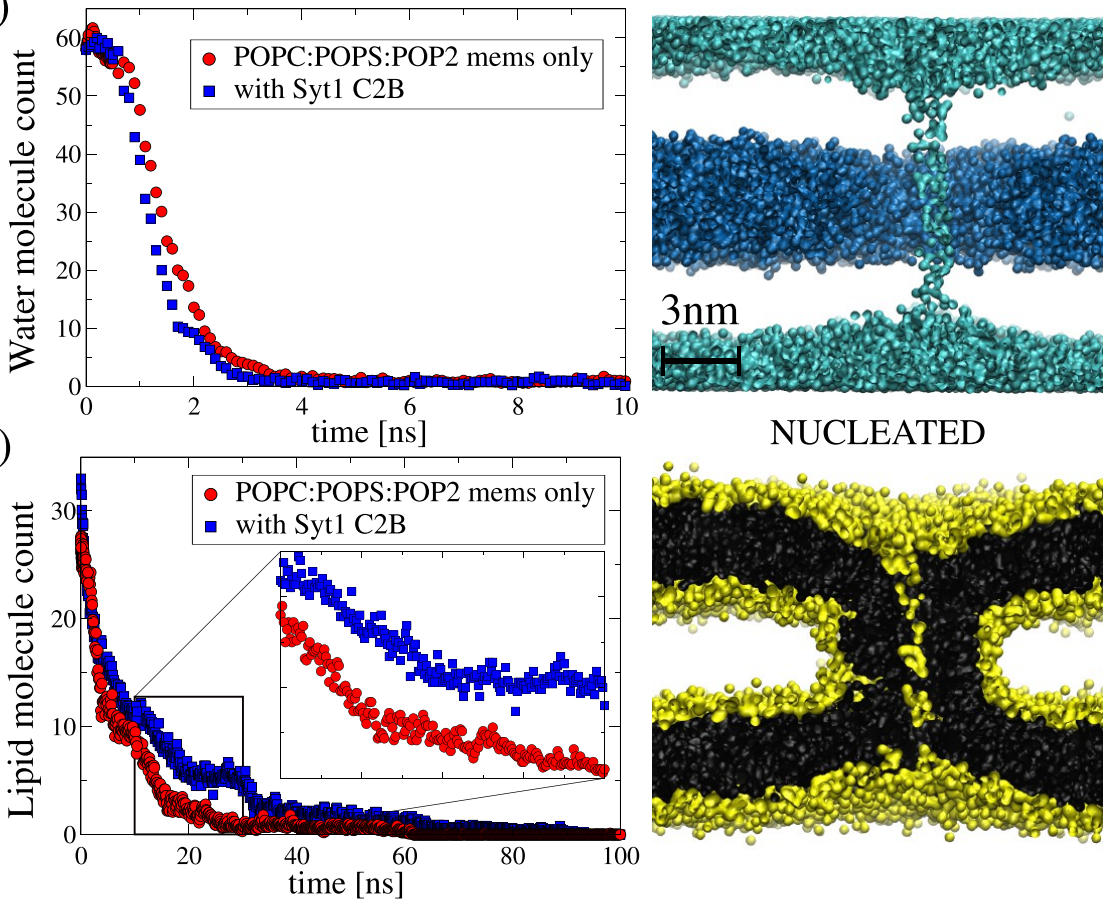

NUCLEATED

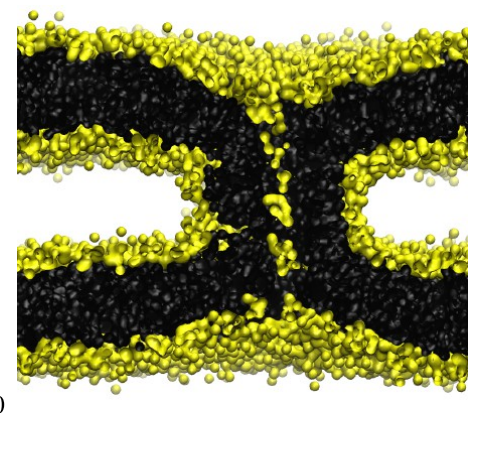

Figure 4. Averaged unbiased molecular dynamics over 10 independent pore-closing trajectories in POPC:POPS:POP2 bilayers starting from the nucleated fusion pore. (a) Water channel molecule count. (b) Count of lipid molecules lining the water channel. Molecular dynamics snapshots show all lipid molecules in black with PO4, NC3, and GL beads highlighted in yellow. Intraorganelle water is cyan, and cytosolic water is blue. Both panels compare the two situations of the presence (blue squares) and absence (red circles) of the synaptotagmin-1 C2B domain.

process $\left(0.7<\xi^{\prime}<1\right)$; see the Supporting Information for polynomial fittings. Figure $3 \mathrm{~b}, \mathrm{c}$ shows the final stages of the fusion pore during stalk hemifusion $\left(0.7<\xi^{\prime}<0.9\right)$ and thin water-channel formation $\left(0.9<\xi^{\prime}<1\right)$. Panel b shows lipid molecules only, and c shows water molecules only. See the Supporting Information for convergence and technical details on umbrella sampling simulations for this free-energy profile.

Hysteresis-Free Sampling. Potential of mean force (PMF) calculations allow for a quantitative estimation of the free-energy surface projected along some reaction coordinate. The reaction coordinate(s) used for biasing sampling must contain all the free-energy barriers that separate states of interest, and the free-energy profile must be independent of the direction of the reaction taking place between these states. ${ }^{81}$ Any difference in free energies along different path directions (i.e., pore opening or pore closing) is an indication of hysteresis, inadequate sampling, or poor convergence. ${ }^{9}$ Although the methodology proposed by Hub and Awasthi ${ }^{8}$ has proven to be hysteresis-free, we have nevertheless tested our $\xi^{\prime}$ implementation of the concentric cylinders to ensure adequate sampling.

Accordingly, we have run PMF calculations in both directions: forward from $\xi^{\prime} \approx 0.2$ (planar and parallel bilayers) to $\xi^{\prime} \approx 1$ (nucleated fusion pore) and backward. Figure 3a shows free-energy profiles for two independent sets of 31 windows of $100 \mathrm{~ns}$ each following pore-opening (black line) and pore-closing (red line) paths. Initial configurations were taken from over $100 \mathrm{~ns}$ slow-growth, pore-opening and poreclosing simulations, as originally suggested by Pearlman and Kollman. ${ }^{82}$ It can be observed that hysteresis is not significant between paths (see panel a). For convergence and technical details on umbrella sampling simulations, see the Supporting Information.
The Synaptotagmin-1 C2B Domain Plays No Significant Role during Fusion Pore Nucleation. To address the problem of describing the effects of the synaptotagmin-1 C2B domain during fusion pore formation, we applied our computational methodology in a system with biologically relevant membranes. The chosen bilayers include 1-palmitoyl2-oleoyl-glycero-3-phosphocholine (POPC), 1-palmitoyl-2oleoyl-sn-glycero-3-phospho-L-serine (POPS), and the MARTINI POP2 general model for phosphatidylinositol bisphosphate lipids, corresponding to the atomistic model C16:1(9c),C18:1(9c) dioleoyl (DO-PIP 2$){ }^{83-86}$ This membrane composition (POPC:POPS:POP2) in a concentration of 87.5:10:2.5 follows the experimental arrangement proposed by Jahn and co-workers ${ }^{87}$ to trap synaptotagmin-1 in the plasma membrane in the presence of calcium.

First, to search for any hidden free-energy barrier in the phase space of $\xi^{\prime}$, we have conducted 10 independent unbiased simulations of $100 \mathrm{~ns}$ each, starting from the nucleated fusion pore between POPC:POPS:POP2 bilayers. Figure 4 shows the averaged evolution of the water channel and the hemifusion lipids as the pore shrinks and closes in the presence (blue squares) and absence (red circles) of the synaptotagmin-1 C2B domain. It can be observed that, when the nucleated fusion pore is released from restrains, it effectively closes and its water channel disconnects after 4 or 5 ns (see panel a). Also, lipid molecules lining the water channel rapidly reorganize for membrane recovery in $t \approx 40 \mathrm{~ns}$ (see panel b). For each molecular dynamics frame, we counted the number of water and lipid molecules between the bilayers using as upper and lower limits the $\mathrm{Z}$ coordinates of the planes containing PO4 lipid beads at opposite leaflets. See the Supporting Information for plots of each one of the $100 \mathrm{~ns}$ pore-closing unbiased simulations. 
In agreement with these findings, Figure 5 shows the freeenergy profiles to open a fusion pore between POPC:POP-

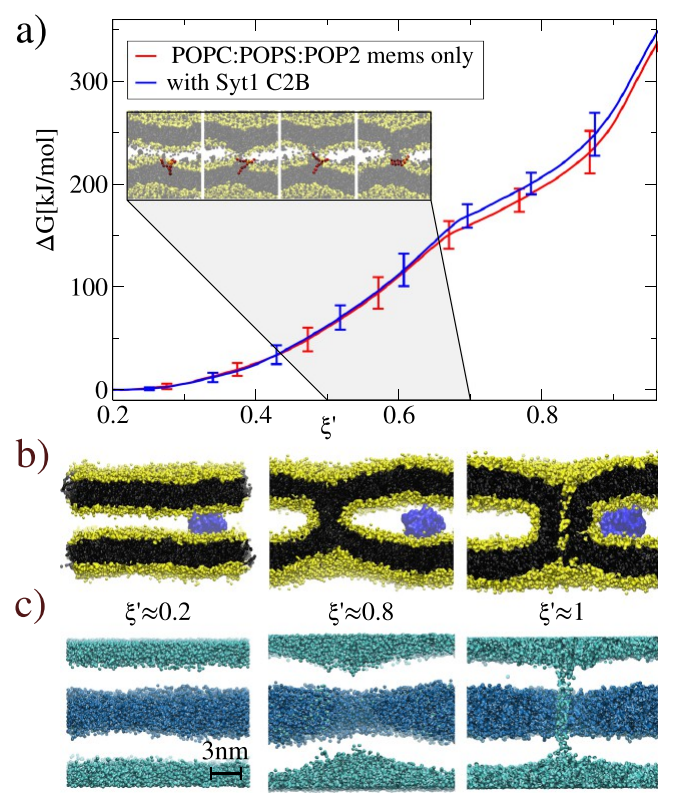

Figure 5. Synaptotagmin-1 C2B domain effects on the energetics of fusion pore nucleation. (a) Free-energy profiles for fusion pore nucleation between two POPC:POPS:POP2 (87.5:10:2.5) bilayers only (red) and with one molecule of synaptotagmin-1 C2B (blue). Error bars are standard errors calculated by individually splitting the profiles in independent blocks. Molecular dynamics insets highlight in red a single POPS lipid while tilting and splaying $\left(0.5<\xi^{\prime}<0.7\right)$, leading to the hemifusion stage. (b) Side view of molecular dynamics snapshots of lipid molecules only with the Synaptotagmin-1 C2B domain between bilayers (dark blue). All lipid molecules are black with PO4, NC3, and GL beads highlighted in yellow. (c) Side view of water molecules only for the same snapshots shown in (b) with intraorganelle water in cyan and cytosolic water in blue.
S:POP2 lipid bilayers only (red line) and with the synaptotagmin-1 C2B domain (blue line) with a total cost of $\sim 350 \mathrm{~kJ} / \mathrm{mol}$ in both cases. Importantly, panel a shows that, from $\xi^{\prime} \approx 1$ to $\xi^{\prime} \approx 0.2$, free energy monotonically goes down, verifying the absence of an energy barrier, as observed before for the unbiased simulations in Figure 4. Noticeably, freeenergy profiles in panel a indicate that the synaptotagmin-1 C2B domain has no significant effects on the fusion pore nucleation process (neither stalk hemifusion nor thin waterchannel formation).

The Synaptotagmin-1 C2B Domain Induces POP2 Clusters to Stabilize the Fusion Pore. Analogous to the experimental literature on fusion pore stabilization, ${ }^{88-92}$ we have here evaluated the stability of the fusion pore in terms of its lifetime. Interestingly, we have found that the expanded fusion pore struggles with an energy barrier (beyond the phase space of $\xi^{\prime}$ ) that prevents it from closing. Again, we run 10 independent unbiased molecular dynamics simulations of 100 ns each, this time starting from an expanded fusion pore in two cases: POPC:POPS:POP2 bilayers only and with the synaptotagmin-1 $\mathrm{C} 2 \mathrm{~B}$ domain. In Figure $6 \mathrm{a}, \mathrm{b}$, averaged trajectories are shown for each one of the two systems, plotting the amount of water molecules contained in the channel and the amount of lipids around it, respectively. In principle, it can be observed that both amounts of water molecules in the channel and lipid molecules in its surroundings remain high, when compared to Figure 4.

However, the behavior of the fusion pore under unbiased conditions is significantly different for longer simulations times (several $\mu \mathrm{s}$ ). Figure $6 \mathrm{c}$ plots the amount of lipids lining the water channel for the expanded fusion pore in the presence (blue squares) and absence (red circles) of $\mathrm{C} 2 \mathrm{~B}$ domain. Remarkably, the expanded fusion pore remains open under norestrain conditions until $1.1 \mu \mathrm{s}$ for membranes only, while it remains stable for at least $10 \mu \mathrm{s}$ for membranes containing one $\mathrm{C} 2 \mathrm{~B}$ domain.

This unexpected difference of 1 order of magnitude is clarified by describing protein-lipid interactions at the

a)
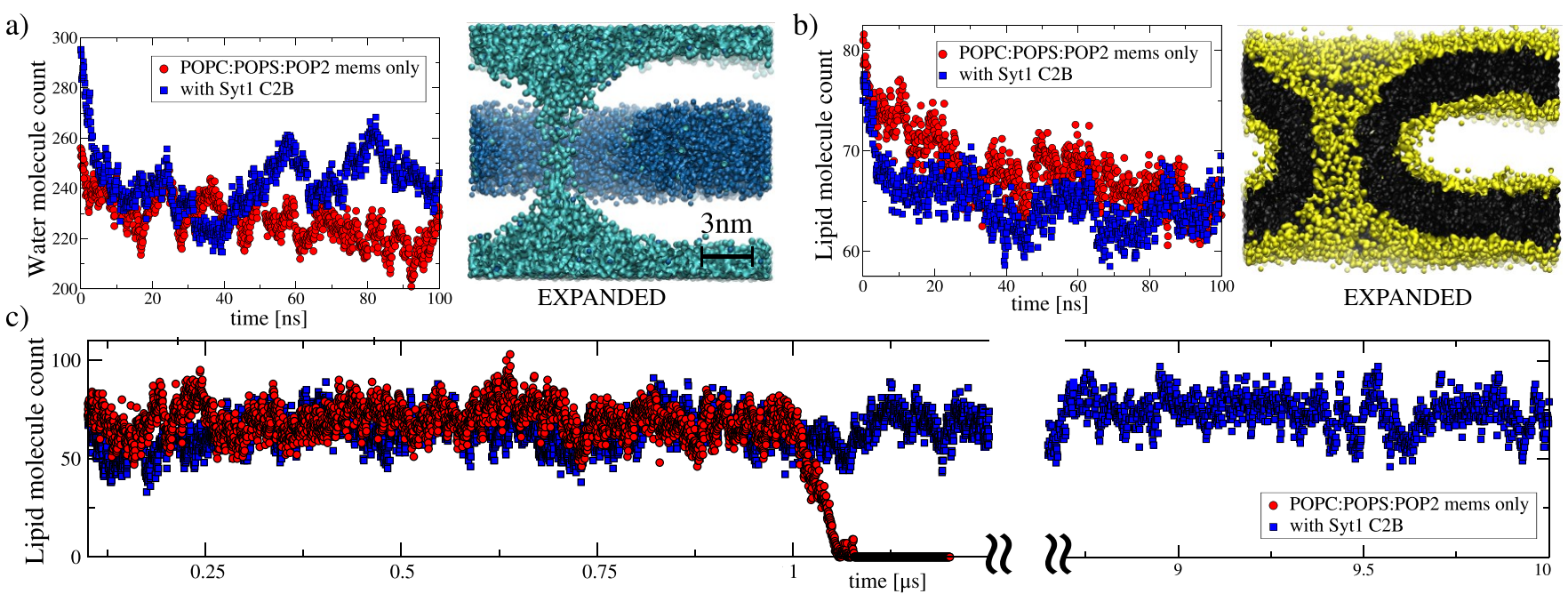

Figure 6. Averaged unbiased molecular dynamics over 10 independent pore closing trajectories in POPC:POPS:POP2 bilayers starting from an expanded fusion pore. (a) Water channel molecule count. (b) Count of lipid molecules lining the water channel. Molecular dynamics snapshots show all lipid molecules in black with PO4, NC3, and GL beads highlighted in yellow. Intraorganelle water is cyan, and cytosolic water is blue. (c) Lipid molecule count in the $\mu$ s time scale to observe definitive pore closure times. All panels compare both situations of the presence (blue squares) and absence (red circles) of the synaptotagmin-1 C2B domain. 

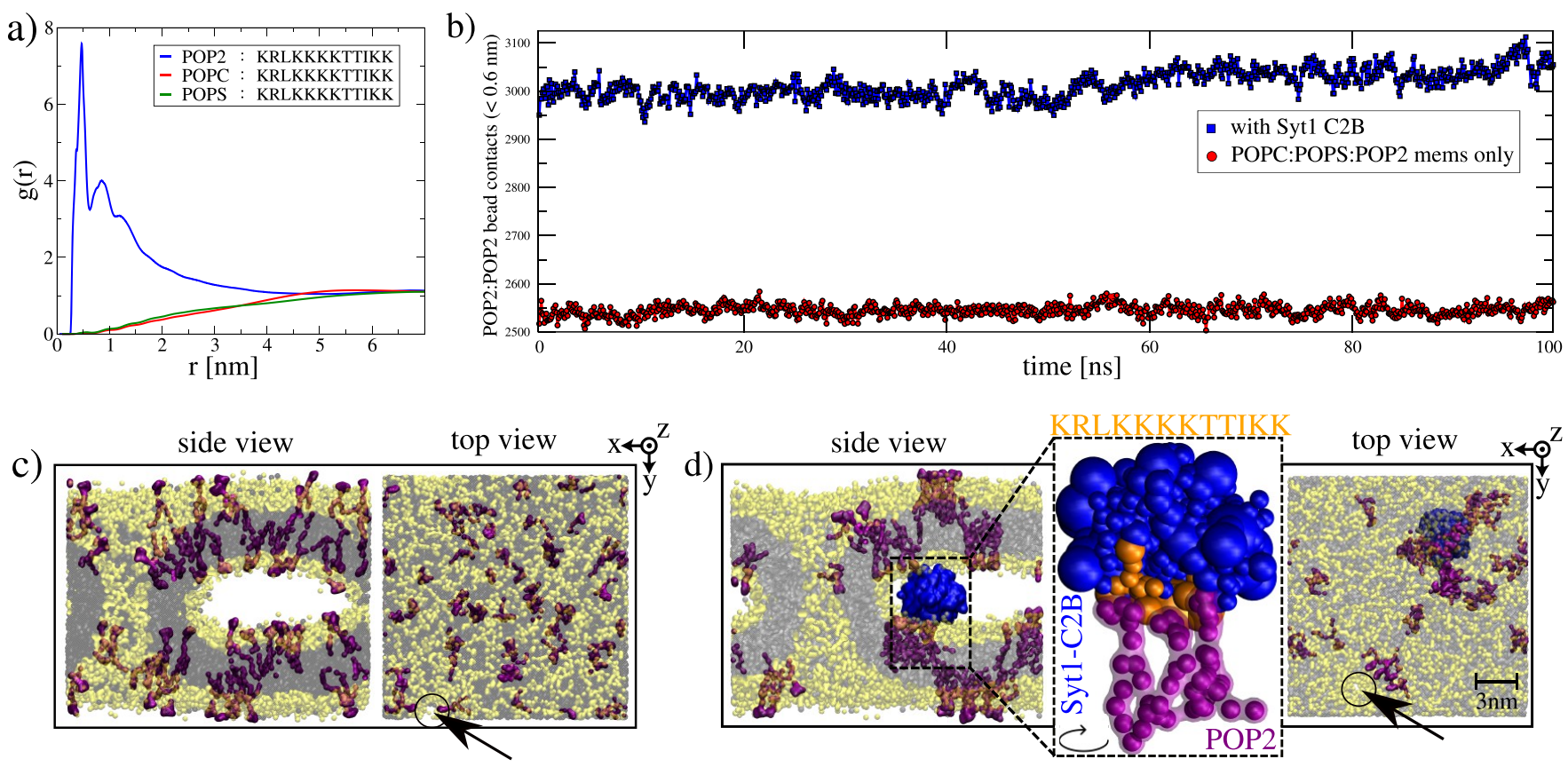

Figure 7. Quantification of $\mathrm{C} 2 \mathrm{~B}$ domain effects on POP2 lipids. (a) Radial distribution functions for POP2, POPC, and POPS with KRLKKKKTTIKK. (b) Ensemble average of POP2:POP2 contacts $(<0.6 \mathrm{~nm})$ over 10 unbiased independent simulations for the standing expanded fusion pore for membranes only (red circles) and with the C2B domain (blue squares). (c, d) Molecular dynamics snapshots of the fusion pore, highlighting POP2 lipids in purple with the C2B domain in blue and the polybasic region in orange. Other lipid molecules are black with PO4, NC3, and GL beads colored yellow. Black arrows indicate the water channel XY position. Water molecules are not shown for better clarity.

molecular level between different groups of protein and lipid molecules. In Figure $7 \mathrm{a}$, we have used a radial distribution function $g(r)$ to quantify polybasic region coordination with the three species of lipids in the bilayers (POPC, POPS, and POP2) as a function of the distance $(r)$. Remarkably, most POP2 lipids are in the neighborhood $(\sim 0.6 \mathrm{~nm})$ of the polybasic region, meaning that KRLKKKKTTIKK highly coordinates with POP2 lipids.

As a practical measure of POP2 clustering, Figure $7 \mathrm{~b}$ shows the POP2:POP2 number of bead contacts $(<0.6 \mathrm{~nm})$ averaged over 10 independent unbiased simulations for the standing expanded fusion pore. It can be observed that the fusion pore with membranes only shows a significantly lower amount of POP2:POP2 contacts (red circles), while POP2 selfinteractions are systematically increased in the presence of the $\mathrm{C} 2 \mathrm{~B}$ domain (blue squares), as already suggested by the radial distribution function in panel a (blue line). This result points to POP2 lipid clusters as the key to any difference in fusion pore dynamics, explaining the longer lifetime of the fusion pore as shown in Figure $6 \mathrm{c}$ and in agreement with experimental results. ${ }^{30-32,46,48}$

Molecular dynamics snapshots in Figure $7 \mathrm{c}, \mathrm{d}$ graphically describe the POP2 spatial distribution in the membranes. Clear clusters can be observed due to KRLKKKKTTIKK:POP2 enhanced interactions (see panel d) and practically no clusters for membranes only (see panel c). Radial distribution functions $g(r)$ in panel a were calculated along the first $1 \mu \mathrm{s}$ of unbiased molecular dynamics, starting from the expanded fusion pores to represent the dynamics of the protein-lipid systems while the fusion pores are open and under no restrain.

The Lysine-Rich Polybasic Patch Selectively Interacts with POP2 Lipids. As phosphatidylinositol-4,5-bisphosphate lipid clusters have been reported to facilitate membrane bending, ${ }^{45,49-51}$ KRLKKKKTTIKK strong binding to POP2 lipids suggests these interactions to be one of the possible molecular mechanisms used by the synaptotagmin-1 C2B domain to keep membranes from returning to their original planar shape, therefore being one of the reasons for fusion pore stabilization. We have verified POP2 clustering and fusion pore stabilization by POP2:KRLKKKKTTIKK binding in other bilayers at different POP2 concentrations. For details, see Table S3 and Figure S11 in the Supporting Information.

Furthermore, we have conducted the same unbiased simulations starting from an expanded fusion pore, this time with POP2 lipids entirely replaced by phosphatidylethanolamines (POPE) keeping the original (87.5:10:2.5) concentrations. Radial distribution functions show that POPE lipids do not bind significantly to the KRLKKKKTTIKK polybasic region, and measurements of POPE:POPE contacts $(<0.6 \mathrm{~nm})$ show that POPE lipids have no propensity to form clusters neither with nor without the synaptotagmin-1 C2B domain. For details, see Figure S12 in the Supporting Information.

To support our conclusion, we have reversed coarse-grained configurations of the stabilized fusion pore to an all-atom representation (using the CHARMM-GUI all-atom convert$\mathrm{er}^{93}$ ). Figure 8a shows an expanded fusion pore released from restrain and after $10 \mu$ s of unbiased molecular dynamics before being converted to an all-atom representation. Panel $b$ shows the specific interactions between the polybasic region KRLKKKKTTIKK lysine residues in C2B and strongly anionic $\mathrm{PIP}_{2}$ lipids of the binding cluster. $\mathrm{PIP}_{2}$ has a negative net charge and easily interacts with polybasic stretches of amino acids. ${ }^{39,46}$ Residues K321, K324, K325, K326, and K327 establish stable interactions with $\mathrm{PIP}_{2}$ phosphate groups, inducing their aggregation around the polybasic region during the molecular dynamics trajectory. 
a)

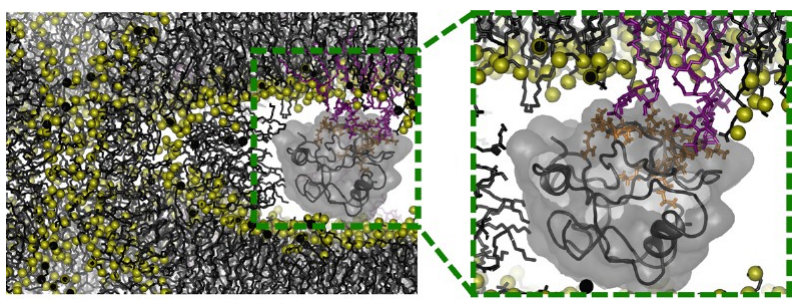

b)

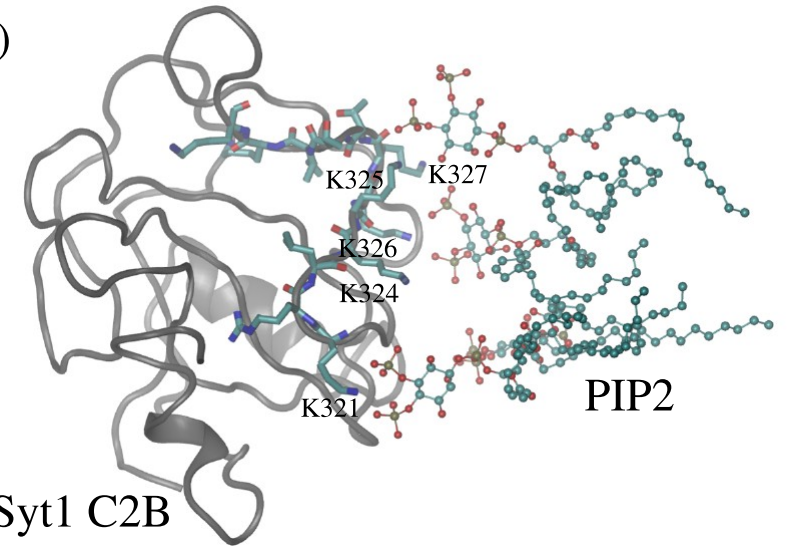

Figure 8. Atomistic view of the fusion pore and $\mathrm{PIP}_{2}$ clustering around the polybasic region KRLKKKKTTIKK in the synaptotagmin$1 \mathrm{C} 2 \mathrm{~B}$ domain. (a) Details of the stabilized fusion pore for atomistic POPC:POPS:PIP 2 bilayers with the atomistic C2B domain. The polybasic region is highlighted in orange sticks, and one $\mathrm{PIP}_{2}$ lipid cluster is colored in purple sticks. Yellow spheres are $\mathrm{P}$ and $\mathrm{N}$ atoms in POPC and POPS lipids, and the rest of the lipids are in black sticks. Water molecules are not shown. (b) Protein-lipid interactions between the polybasic region and the $\mathrm{PIP}_{2}$ lipid cluster. $\mathrm{PIP}_{2}$ lipids are in balls and sticks, and amino acids in the polybasic region are sticks; both follow the Corey-Pauling-Koltun (CPK) coloring convention for distinguishing atoms. Gray ribbons show the entire C2B domain.

\section{CONCLUSIONS}

We have explored the phase space of fusion pore dynamics along (i) membrane bending, (ii) membrane fusion, and (iii) nucleation using a specifically designed reaction coordinate. We have demonstrated that the synaptotagmin-1 C2B domain has negligible effects on the nucleation of the fusion pore. However, by expanding the fusion pore, we have shown that the $\mathrm{C} 2 \mathrm{~B}$ polybasic lysine-rich region strongly interacts with highly anionic phosphatidylinositol bisphosphate lipid molecules, driving the formation of POP2 aggregates and extending the life of the fusion pore. Though, we have quantitatively observed that the polybasic region KRLKKKKTTIKK in C2B masters the decisive protein-lipid interactions that induce the formation of POP2 clusters, which collectively stabilizes the expanded fusion pore.

\section{COMPUTATIONAL METHODS}

We conducted all our simulations with GROMACS$2018.3^{94-96}$ under the MARTINI coarse-grained model, ${ }^{86}$ which is widely used for protein-lipid molecular modeling. ${ }^{6,23,97-102}$ In spite of the reduced resolution, MARTINI coarse-grained molecular dynamics successfully captures most of the basic characteristics of many biological systems, allowing for good characterization of complex coupled effects in manybody systems, such as large membranes with protein domains where lipid reorganization is a key problem. ${ }^{86,103-107}$
MARTINI groups four to six non-hydrogen atoms into a single particle, which interact following a parameterization that reproduces experimental data with sufficient accuracy to observe large conformational changes, such as the formation of the fusion pore. All simulation systems used here consisted of two bilayers of 1024 lipid molecules each. This size of bilayers ensures negligible finite-size effects due to interactions between periodic images. ${ }^{8}$ For each pair of bilayers, at least 15 coarse-grained waters per lipid molecule were included (ample water condition for MARTINI). ${ }^{84}$ In all cases, we used the polarizable water model for MARTINI. ${ }^{108}$ We have conducted our simulations using the MARTINI 2.2 force field which, as recently pointed out, ${ }^{109}$ is being reparameterized into MARTINI 3 to improve interactions between phospholipid bilayers and proteins.

Simulation Setup. All production molecular dynamics simulations were run in the semi-isotropic NPT ensemble at $T$ $=303.15 \mathrm{~K}^{110-114}$ using the V-rescale thermostat ${ }^{115}$ with a coupling constant of 1 ps. For pure DPPC bilayers, simulations were also performed at $323 \mathrm{~K}$ (well above the phase transition temperature, $314 \mathrm{~K}$, for $\mathrm{DPPC}^{116,117}$ ); see the Supporting Information for the free-energy profile at this temperature. For equilibration runs, the pressure was set at 1.0 bar using the Berendsen barostat ${ }^{118}$ with a 5 ps coupling constant. For production runs, a Parrinello-Rahman barostat was used instead with a time constant of 12 ps. In all cases, the compressibility was set to $3 \times 10^{-4} \mathrm{bar}^{-1}$, and long-range reaction field electrostatic interactions were used. In addition, as recommended for MARTINI, a time step of $20 \mathrm{fs}$ was set in all cases. Before production runs, all systems were minimized and equilibrated for at least $100 \mathrm{~ns}$ to generate properly relaxed initial configurations.

Radial distribution functions were calculated with GROMACS built-in gmx rdf, and POP2:POP2 interactions were measured with gmx mindist. Coarse-grained bilayers and proteins were prepared in part using the CHARMM-GUI web server. ${ }^{93}$ Molecular dynamics snapshots were visualized using visual molecular dynamics (VMD) ${ }^{119}$ and the academic version of Maestro molecular modeling environment. ${ }^{120}$ Data graphics were plotted with GRACE (Graphing, Advanced Computation and Exploration of data). Figure panels were organized using Inkscape and GIMP (GNU Image Manipulation Program).

PMF Calculations. For DPPC simulations, PMF profiles for inducing the fusion pore were computed with umbrella sampling ${ }^{58,59}$ using a set of 31 windows to span two simultaneous reaction coordinates $\xi_{1}^{\prime}$ and $\xi_{2}^{\prime}$ in the interval $\sim\left[\begin{array}{ll}0.2 & 1\end{array}\right]$. Umbrella windows were unevenly distributed to intensify sampling in regions requiring more detailed descriptions, i.e., lipid hemifusion $\left(0.6<\xi^{\prime}<0.9\right)$ and waterchannel formation $\left(0.9<\xi^{\prime}<1\right)$. DPPC bilayers were used here as a study system to test the reaction coordinate and check for hysteresis-free sampling.

For POPC:POPS:POP2 bilayers, 22 windows were used to span reaction coordinates $\xi_{1}^{\prime}$ and $\xi_{2}^{\prime}$ in the interval of $\sim[0.21]$. In all cases, harmonic potentials of the form $V_{0}\left(\xi^{\prime}\right)=\frac{1}{2} k\left(\xi^{\prime}-\xi_{0}^{\prime}\right)^{2}$ were applied to simultaneously restrain dynamics with respective force constants $k_{1}$ and $k_{2}$ (set to $20,000 \mathrm{~kJ} / \mathrm{mol}$ for most windows; see the Supporting Information for details). All umbrella windows run for 100 ns, discarding the first 5 ns of each trajectory to allow for equilibration. 
Free-energy profiles were recovered using the weighted histogram analysis method (WHAM) ${ }^{121}$ built-in in GROMACS as gmx wham, ${ }^{66}$ which simultaneously processes both $\xi_{1}^{\prime}$ and $\xi_{2}^{\prime}$ and projects the free-energy profile in the $\xi^{\prime}$ phase space. To double-check the PMF calculations, we computed the free-energy surfaces using a WHAM 2D implementation by Grossfield $^{67}$ (see 2D plots for POPC:POPS:POP2 bilayers in the Supporting Information). Convergence was assessed by repeating these calculations on consecutive trajectory blocks. For technical details on umbrella sampling window distributions and force constant values, see the Supporting Information.

For technical details on the implementation of $\xi^{\prime}$, see the Supporting Information, and for an exhaustive description of the original single cylinder reaction coordinate $(\xi)$, see the work of Hub and Awasthi. ${ }^{8}$ An interesting approach would be to use some parameters of the reaction coordinate $\xi^{\prime}$ (such as $R_{\text {cyl1 }}$ and/or $R_{\text {cyl2 }}$ ) to bias the fusion pore expansion and estimate the associated free-energy profile; however, our efforts in this direction led to hysteresis problems.

$\xi$ Reaction Coordinate. Reaction coordinate $\xi$ as implemented by Hub and Awasthi ${ }^{8}$ in GROMACS is capable of inducing the formation of a hydrophilic pore in a single lipid bilayer. The reaction coordinate shows virtually no hysteresis and uses a membrane spanning cylinder that is decomposed into slices along the membrane normal. The cylinder is defined by the number of cylinder slices $\left(N_{s}\right)$, its thickness $\left(d_{s}\right)$, its radius $\left(R_{\text {cyl }}\right)$, and its occupation factor $(\zeta)$; see eqs $2-4$. In Hub and Awasthi's work, the reaction coordinate is defined as the fraction of slices that is occupied by polar heavy atoms, namely, water oxygen atoms as well as the four oxygen atoms of the lipid phosphate groups. ${ }^{8}$ This definition of the reaction coordinate makes not only water molecules enter the membrane but also the surrounding lipids tilt and accommodate to the forming water channel. The result is a welldefined hydrophilic transmembrane pore containing a thin water channel.

$$
\xi=\frac{1}{N_{s}} \sum_{s=0}^{N_{s}-1} \delta_{s}\left(N_{s}^{(p)}\right)
$$

Here, $N_{s}^{(p)}$ accounts for the number of beads within slice $s$ inside the membrane-spanning cylinder. $\delta_{s}$ is a continuous function ranging from 0 (for no beads in slice $s$ ) to 1 (for 1 or more beads in slice $s$ ) as defined in eq 3 .

$$
\delta_{s}\left(N_{s}^{(p)}\right)=\psi\left(N_{s}^{(p)} ; \zeta\right)
$$

The switching piecewise-defined function for $\xi$ is linearexponential, continuous, and differentiable, as shown in eq 4.

$$
\psi(x ; \zeta)=\left\{\begin{array}{lll}
\zeta x & \text { if } & x \leq 1 \\
1-c e^{-b x} & \text { if } & x>1
\end{array}\right.
$$

Parameters $b$ and $c$ are defined as a function of the occupation factor $\zeta$ (see eqs 5 and 6 ).

$$
\begin{aligned}
& b=\frac{\zeta}{1-\zeta} \\
& c=(1-\zeta) e^{b}
\end{aligned}
$$

For simplicity and to acknowledge Hub's work, we kept the same nomenclature in our modified reaction coordinate. For a complete mathematical description, please refer to the original publication. $^{8}$

\section{ASSOCIATED CONTENT}

\section{(s) Supporting Information}

The Supporting Information is available free of charge at https://pubs.acs.org/doi/10.1021/acs.jctc.0c00734.

PMF convergence analysis, histograms, and umbrella sampling details for free-energy profiles; polynomial fitting of the DPPC free-energy profile; a free-energy surface using WHAM-2D for both biasing potentials during fusion pore nucleation; unbiased simulations for fusion pore closure; molecular dynamics snapshots of the fusion pore with $35 \%$ more cytosolic water molecules; free-energy profile for pore opening between DPPC bilayers at $T=323 \mathrm{~K}$; and expanded fusion pore stabilization for different concentrations of POP2 lipids and for POP2 replaced by POPE at the original concentration (PDF)

\section{AUTHOR INFORMATION}

\section{Corresponding Author}

Diego Masone - Instituto de Histología y Embriología de Mendoza (IHEM) - Consejo Nacional de Investigaciones Científicas y Técnicas (CONICET) and Facultad de Ingeniería, Universidad Nacional de Cuyo (UNCuyo), Mendoza 5500, Argentina; 이이.org/0000-0001-57708614; Phone: +54 261405 4843; Email: diego.masone@ ingenieria.uncuyo.edu.ar

\section{Authors}

Marcelo Caparotta - Facultad de Ciencias Exactas y Naturales, Universidad Nacional de Cuyo (UNCuyo), Mendoza 5500, Argentina

Claudia N. Tomes - Facultad de Ciencias Exactas y Naturales and Instituto de Histología y Embriología de Mendoza (IHEM) - Consejo Nacional de Investigaciones Científicas y Técnicas (CONICET), Universidad Nacional de Cuyo (UNCuyo), Mendoza 5500, Argentina

Luis S. Mayorga - Facultad de Ciencias Exactas y Naturales and Instituto de Histología y Embriología de Mendoza (IHEM) - Consejo Nacional de Investigaciones Científicas y Técnicas (CONICET), Universidad Nacional de Cuyo (UNCuyo), Mendoza 5500, Argentina

Complete contact information is available at: https://pubs.acs.org/10.1021/acs.jctc.0c00734

\section{Notes}

The authors declare no competing financial interest.

\section{ACKNOWLEDGMENTS}

The supercomputing time for this work was provided by the Sistema Nacional de Computación de Alto Desempeño (SNCAD), Iniciativa de Proyectos Acelerados de Cálculo (IPAC), grant nos. 2017-75-APN-SECACT\#MCT, 2018-6APN-SECACT\#MECCYT, and 2019-72-APN-SECACT\#MECCYT. Also, grants from CONICET (no. PIP-13CO01), ANPCyT (no. PICT2017-1002), and GPU hardware by the NVIDIA Corporation are gratefully acknowledged. The authors thank Prof. Jochen Hub for generously sharing his reaction coordinate source code. 


\section{REFERENCES}

(1) Wohlert, J.; den Otter, W. K.; Edholm, O.; Briels, W. J. Free energy of a transmembrane pore calculated from atomistic molecular dynamics simulations. J. Chem. Phys. 2006, 124, 154905.

(2) Tolpekina, T. V.; den Otter, W. K.; Briels, W. J. Nucleation free energy of pore formation in an amphiphilic bilayer studied by molecular dynamics simulations. J. Chem. Phys. 2004, 121, 12060.

(3) Tieleman, D. P.; Leontiadou, H.; Mark, A. E.; Marrink, S.-J. Simulation of Pore Formation in Lipid Bilayers by Mechanical Stress and Electric Fields. J. Am. Chem. Soc. 2003, 125, 6382-6383.

(4) Fuertes, G.; Giménez, D.; Esteban-Martín, S.; Sánchez-Muñoz, O. L.; Salgado, J. A lipocentric view of peptide-induced pores. Eur. Biophys. J. 2011, 40, 399-415.

(5) Bennett, W. F. D.; Sapay, N.; Tieleman, D. P. Atomistic Simulations of Pore Formation and Closure in Lipid Bilayers. Biophys. J. 2014, 106, 210-219.

(6) Sharma, S.; Lindau, M. Molecular mechanism of fusion pore formation driven by the neuronal SNARE complex. Proc. Natl. Acad. Sci 2018, 115, 12751-12756.

(7) Mirjalili, V.; Feig, M. Density-biased sampling: a robust computational method for studying pore formation in membranes. J. Chem. Theory Comput. 2015, 11, 343-350.

(8) Hub, J. S.; Awasthi, N. Probing a Continuous Polar Defect: A Reaction Coordinate for Pore Formation in Lipid Membranes. J. Chem. Theory Comput. 2017, 13, 2352-2366.

(9) Awasthi, N.; Hub, J. S. Simulations of Pore Formation in Lipid Membranes: Reaction Coordinates, Convergence, Hysteresis, and Finite-Size Effects. J. Chem. Theory Comput. 2016, 12, 3261-3269.

(10) Blokhuis, E. M.; D’Agostino, M.; Mayer, A.; Risselada, H. J. Fusion Pores Live on the Edge. J. Phys. Chem. Lett. 2020, 11, 12041208.

(11) Brunger, A. T.; Choi, U. B.; Lai, Y.; Leitz, J.; Zhou, Q. Molecular Mechanisms of Fast Neurotransmitter Release. Annu. Rev. Biophys. 2018, 47, 469-497.

(12) Chang, C.-W.; Chiang, C.-W.; Jackson, M. B. Fusion pores and their control of neurotransmitter and hormone release. J. Gen. Physiol. 2017, 149, 301-322.

(13) Wu, Z.; Dharan, N.; Thiyagarajan, S.; O'Shaughnessy, B.; Karatekin, E. The neuronal calcium sensor Synaptotagmin-1 and SNARE proteins cooperate to dilate fusion pores mechanically. bioRxiv 2019, 623827.

(14) Hui, S.; Stewart, T.; Boni, L.; Yeagle, P. Membrane fusion through point defects in bilayers. Science 1981, 212, 921-923.

(15) Siegel, D. P. Inverted micellar intermediates and the transitions between lamellar, cubic, and inverted hexagonal lipid phases II. Implications for membrane-membrane interactions and membrane fusion. Biophys. J. 1986, 49, 1171-1183.

(16) Noguchi, H. Fusion and toroidal formation of vesicles by mechanical forces: A Brownian dynamics simulation. J. Chem. Phys. 2002, 117, 8130-8137.

(17) Risselada, H. J.; Marelli, G.; Fuhrmans, M.; Smirnova, Y. G.; Grubmüller, H.; Marrink, S. J.; Müller, M. Line-Tension Controlled Mechanism for Influenza Fusion. PLoS One 2012, 7, 1-14.

(18) Kuzmin, P. I.; Zimmerberg, J.; Chizmadzhev, Y. A.; Cohen, F. S. A quantitative model for membrane fusion based on low-energy intermediates. Proc. Natl. Acad. Sci. U. S. A. 2001, 98, 7235-7240.

(19) Siegel, D. P. The Gaussian curvature elastic energy of intermediates in membrane fusion. Biophys. J. 2008, 95, 5200-5215.

(20) Daoulas, K. C.; Müller, M. Exploring thermodynamic stability of the stalk fusionintermediate with three-dimensional self-consistent field theory calculations. Soft Matter 2013, 9, 4097-4102.

(21) Grafmüller, A.; Shillcock, J.; Lipowsky, R. The fusion of membranes and vesicles: pathway and energy barriers from dissipative particle dynamics. Biophys. J. 2009, 96, 2658-2675.

(22) Müller, M.; Smirnova, Y. G.; Marelli, G.; Fuhrmans, M.; Shi, A.C. Transition Path from Two Apposed Membranes to a Stalk Obtained by a Combination of Particle Simulations and String Method. Phys. Rev. Lett. 2012, 108, 228103.
(23) Smirnova, Y. G.; Risselada, H. J.; Müller, M. Thermodynamically reversible paths of the first fusion intermediate reveal an important role for membrane anchors of fusion proteins. Proc. Natl. Acad. Sci 2019, 116, 2571-2576.

(24) Geppert, M.; Goda, Y.; Hammer, R. E.; Li, C.; Rosahl, T. W.; Stevens, C. F.; Südhof, T. C. Synaptotagmin I: A major Ca2+ sensor for transmitter release at a central synapse. Cell 1994, 79, 717-727.

(25) Littleton, J. T. A Genomic Analysis of Membrane Trafficking and Neurotransmitter Release in Drosophila. J. Cell Biol. 2000, 150, $77-82$.

(26) Bradberry, M. M.; Bao, H.; Lou, X.; Chapman, E. R. Phosphatidylinositol 4,5-bisphosphate drives $\mathrm{Ca}^{2+}$-independent membrane penetration by the tandem $\mathrm{C}_{2}$ domain proteins synaptotagmin-1 and Doc2 $\beta$. J. Biol. Chem. 2019, 10942.

(27) Chapman, E. R.; Davis, A. F. Direct Interaction of a Ca2+binding Loop of Synaptotagmin with Lipid Bilayers. J. Biol. Chem. 1998, 273, 13995-14001.

(28) Fernández-Chacón, R.; Königstorfer, A.; Gerber, S. H.; García, J.; Matos, M. F.; Stevens, C. F.; Brose, N.; Rizo, J.; Rosenmund, C.; Südhof, T. C. Synaptotagmin I functions as a calcium regulator of release probability. Nature 2001, 410, 41-49.

(29) Rhee, J.-S.; Li, L. Y.; Shin, O.-H.; Rah, J.-C.; Rizo, J.; Sudhof, T. C.; Rosenmund, C. Augmenting neurotransmitter release by enhancing the apparent $\mathrm{Ca} 2+$ affinity of synaptotagmin 1 . Proc. Natl. Acad. Sci. U. S. A. 2005, 102, 18664-18669.

(30) Bai, J.; Tucker, W. C.; Chapman, E. R. PIP2 increases the speed of response of synaptotagmin and steers its membrane-penetration activity toward the plasma membrane. Nat. Struct. Mol. Biol. 2004, 11, 36-44.

(31) Li, L.; Shin, O.-H.; Rhee, J.-S.; Araç, D.; Rah, J.-C.; Rizo, J.; Südhof, T.; Rosenmund, C. Phosphatidylinositol Phosphates as Coactivators of $\mathrm{Ca} 2+$ Binding to $\mathrm{C} 2$ Domains of Synaptotagmin 1. J. Biol. Chem. 2006, 281, 15845-15852.

(32) Radhakrishnan, A.; Stein, A.; Jahn, R.; Fasshauer, D. The Ca2+ affinity of synaptotagmin 1 is markedly increased by a specific interaction of its C2B domain with phosphatidylinositol 4,5bisphosphate. J. Biol. Chem. 2009, 284, 25749-25760.

(33) van den Bogaart, G.; Thutupalli, S.; Risselada, J. H.; Meyenberg, K.; Holt, M.; Riedel, D.; Diederichsen, U.; Herminghaus, S.; Grubmüller, H.; Jahn, R. Synaptotagmin-1 may be a distance regulator acting upstream of SNARE nucleation. Nat. Struct. Mol. Biol. 2011, 18, 805-812.

(34) Fernandez, I.; Araç, D.; Ubach, J.; Gerber, S. H.; Shin, O.-H.; Gao, Y.; Anderson, R. G. W.; Südhof, T. C.; Rizo, J. ThreeDimensional Structure of the Synaptotagmin 1 C2B-Domain: Synaptotagmin 1 as a Phospholipid Binding Machine. Neuron 2001, 32, 1057-1069.

(35) Wang, S.; Li, Y.; Ma, C. Synaptotagmin-1 C2B domain interacts simultaneously with SNAREs and membranes to promote membrane fusion. eLife 2016, 5, No. e14211.

(36) Lai, Y.; Lou, X.; Diao, J.; Shin, Y.-K. Molecular origins of synaptotagmin 1 activities on vesicle docking and fusion pore opening. Sci. Rep. 2015, 5, 9267.

(37) Roggero, C. M.; De Blas, G. A.; Dai, H.; Tomes, C. N.; Rizo, J.; Mayorga, L. S. Complexin/Synaptotagmin Interplay Controls Acrosomal Exocytosis. J. Biol. Chem. 2007, 282, 26335-26343.

(38) Bendahmane, M.; Bohannon, K. P.; Bradberry, M. M.; Rao, T. C.; Schmidtke, M. W.; Abbineni, P. S.; Chon, N. L.; Tran, S.; Lin, H.; Chapman, E. R.; et al. The synaptotagmin C2B domain calciumbinding loops modulate the rate of fusion pore expansion. $\mathrm{MBoC}$ 2018, 29, 834-845.

(39) McLaughlin, S.; Wang, J.; Gambhir, A.; Murray, D. PIP 2 and proteins: interactions, organization, and information flow. Annu. Rev. Biophys. Biomol. Struct. 2002, 31, 151-175.

(40) Morales, K. A.; Igumenova, T. I. Synergistic effect of $\mathrm{Pb}(2+)$ and phosphatidylinositol 4,5-bisphosphate on C2 domain-membrane interactions. Biochemistry 2012, 51, 3349-3360. 
(41) Das, D.; Bao, H.; Courtney, K. C.; Wu, L.; Chapman, E. R. Resolving kinetic intermediates during the regulated assembly and disassembly of fusion pores. Nat. Commun. 2020, 11, 231.

(42) Montaville, P.; Coudevylle, N.; Radhakrishnan, A.; Leonov, A.; Zweckstetter, M.; Becker, S. The PIP2 binding mode of the C2 domains of rabphilin-3A. Protein science : a publication of the Protein Society 2008, 17, 1025-1034.

(43) Coudevylle, N.; Montaville, P.; Leonov, A.; Zweckstetter, M.; Becker, S. Structural determinants for $\mathrm{Ca} 2+$ and phosphatidylinositol 4,5-bisphosphate binding by the $\mathrm{C} 2 \mathrm{~A}$ domain of rabphilin-3A. The Journal of biological chemistry 2008, 283, 35918-35928.

(44) Guerrero-Valero, M.; Marín-Vicente, C.; Gómez-Fernández, J. C.; Corbalán-García, S. The C2 domains of classical PKCs are specific PtdIns(4,5)P2-sensing domains with different affinities for membrane binding. J. Mol. Biol. 2007, 371, 608-621.

(45) Honigmann, A.; van den Bogaart, G.; Iraheta, E.; Jelger Risselada, H.; Milovanovic, D.; Mueller, V.; Müllar, S.; Diederichsen, U.; Fasshauer, D.; Grubmüller, H.; et al. Phosphatidylinositol 4,5bisphosphate clusters act as molecular beacons for vesicle recruitment. Nat. Struct. Mol. Biol. 2013, 20, 679-686.

(46) van den Bogaart, G.; Meyenberg, K.; Risselada, H. J.; Amin, H.; Willig, K. I.; Hubrich, B. E.; Dier, M.; Hell, S. W.; Grubmüller, H.; Diederichsen, U.; et al. Membrane protein sequestering by ionic protein-lipid interactions. Nature 2011, 479, 552-555.

(47) Park, Y.; Seo, J. B.; Fraind, A.; Pérez-Lara, A.; Yavuz, H.; Han, K.; Jung, S.-R.; Kattan, I.; Walla, P. J.; Choi, M.; et al. Synaptotagmin1 binds to PIP2-containing membrane but not to SNAREs at physiological ionic strength. Nat. Struct. Mol. Biol. 2015, 22, 815-823. (48) Wu, Z.; Ma, L.; Zhu, J.; Courtney, N.; Zhang, Y.; Chapman, E. R.; Karatekin, E. A Polybasic Patch on Synaptotagmin-1 C2A Domain is Essential for Evoked Release and Dilation of Fusion Pores. Biophys. J. 2020, 118, 400a.

(49) Shukla, S.; Jin, R.; Robustelli, J.; Zimmerman, Z. E.; Baumgart, T. PIP2 Reshapes Membranes through Asymmetric Desorption. Biophys. J. 2019, 117, 962-974.

(50) McMahon, H. T.; Kozlov, M. M.; Martens, S. Membrane Curvature in Synaptic Vesicle Fusion and Beyond. Cell 2010, 140, 601-605.

(51) Lynch, K. L.; Gerona, R. R. L.; Kielar, D. M.; Martens, S.; McMahon, H. T.; Martin, T. F. J. Synaptotagmin-1 utilizes membrane bending and SNARE binding to drive fusion pore expansion. Mol. Biol. Cell 2008, 19, 5093-5103.

(52) Mu, L.; Tu, Z.; Miao, L.; Ruan, H.; Kang, N.; Hei, Y.; Chen, J.; Wei, W.; Gong, F.; Wang, B.; et al. A phosphatidylinositol 4,5bisphosphate redistribution-based sensing mechanism initiates a phagocytosis programing. Nat. Commun. 2018, 9, 4259.

(53) Park, Y.; Ryu, J.-K. Models of synaptotagmin-1 to trigger Ca2+-dependent vesicle fusion. FEBS Lett. 2018, 592, 3480-3492.

(54) James, D. J.; Khodthong, C.; Kowalchyk, J. A.; Martin, T. F. J. Phosphatidylinositol 4,5-bisphosphate regulates SNARE-dependent membrane fusion. J. Cell Biol. 2008, 182, 355-366.

(55) Salvalaglio, M.; Perego, C.; Giberti, F.; Mazzotti, M.; Parrinello, M. Moleculardynamics simulations of urea nucleation from aqueous solution. Proc. Natl. Acad. Sci. U. S. A. 2014, 112, E6-E14.

(56) Piaggi, P. M.; Valsson, O.; Parrinello, M. Enhancing Entropy and Enthalpy Fluctuations to Drive Crystallization in Atomistic Simulations. Phys. Rev. Lett. 2017, 119, No. 015701.

(57) Tribello, G. A.; Ceriotti, M.; Parrinello, M. A self-learning algorithm for biased molecular dynamics. Proc. Natl. Acad. Sci 2010, 107, 17509-17514.

(58) Torrie, G. M.; Valleau, J. P. Nonphysical sampling distributions in Monte Carlo free-energy estimation: Umbrella sampling. J. Comput. Phys. 1977, 23, 187-199.

(59) Roux, B. The calculation of the potential of mean force using computer simulations. Comput. Phys. Commun. 1995, 91, 275-282.

(60) Laio, A.; Parrinello, M. Escaping free-energy minima. Proc. Natl. Acad. Sci 2002, 99, 12562-12566.
(61) Laio, A.; Gervasio, F. L. Metadynamics: a method to simulate rare events and reconstruct the free energy in biophysics, chemistry and material science. Rep. Prog. Phys. 2008, 71, 126601.

(62) Barducci, A.; Bussi, G.; Parrinello, M. Well-Tempered Metadynamics: A Smoothly Converging and Tunable Free-Energy Method. Phys. Rev. Lett. 2008, 100, No. 020603.

(63) Saladino, G.; Gauthier, L.; Bianciotto, M.; Gervasio, F. L. Assessing the Performance of Metadynamics and Path Variables in Predicting the Binding Free Energies of p38 Inhibitors. J. Chem. Theory Comput. 2012, 8, 1165-1170.

(64) Smirnova, Y. G.; Fuhrmans, M.; Barragan Vidal, I. A.; Müller, $M$. Free-energy calculation methods for collective phenomena in membranes. J. Phys. D: Appl. Phys. 2015, 48, 343001.

(65) Mirjanian, D.; Dickey, A. N.; Hoh, J. H.; Woolf, T. B.; Stevens, M. J. Splaying of aliphatic tails plays a central role in barrier crossing during liposome fusion. J. Phys. Chem.. B 2010, 114, 11061-11068.

(66) Hub, J. S.; de Groot, B. L.; van der Spoel, D. g_wham A Free Weighted Histogram Analysis Implementation Including Robust Error and Autocorrelation Estimates. J. Chem. Theory Comput. 2010, 6, 3713-3720.

(67) Grossfield, A. WHAM: the weighted histogram analysis method. http://membrane.urmc.rochester.edu/wordpress/page_id=126 version, 2.0.9.1 .

(68) Kawamoto, S.; Klein, M. L.; Shinoda, W. Coarse-grained molecular dynamics study of membrane fusion: Curvature effects on free energy barriers along the stalk mechanism. J. Chem. Phys. 2015, 143, 243112.

(69) Jackson, M. B. Minimum membrane bending energies of fusion pores. The Journal of membrane biology 2009, 231, 101-115.

(70) Cohen, F. S.; Melikyan, G. B. The Energetics of Membrane Fusion from Binding, through Hemifusion, Pore Formation, and Pore Enlargement. The Journal of Membrane Biology 2004, 199, 1-14.

(71) Ryham, R. J.; Ward, M. A.; Cohen, F. S. Teardrop shapes minimize bending energy of fusion pores connecting planar bilayers. Phys. Rev. E 2013, 88, No. 062701.

(72) Chizmadzhev, Y. A.; Cohen, F. S.; Shcherbakov, A.; Zimmerberg, J. Membrane mechanics can account for fusion pore dilation in stages. Biophys. J. 1995, 69, 2489-2500.

(73) Nanavati, C.; Markin, V. S.; Oberhauser, A. F.; Fernandez, J. M. The exocytotic fusion pore modeled as a lipidic pore. Biophys. J. 1992, $63,1118-1132$.

(74) Cunill-Semanat, E.; Salgado, J. Spontaneous and Stress-Induced Pore Formation in Membranes: Theory, Experiments and Simulations. J. Membr. Biol. 2019, 252, 241-260.

(75) Hu, Y.; Sinha, S. K.; Patel, S. Investigating Hydrophilic Pores in Model Lipid Bilayers Using Molecular Simulations: Correlating Bilayer Properties with Pore-Formation Thermodynamics. Langmuir : the ACS journal of surfaces and colloids 2015, 31, 6615-6631.

(76) Jambeck, J. P. M.; Lyubartsev, A. P. Another Piece of the Membrane Puzzle: Extending Slipids Further. J. Chem. Theory Comput. 2013, 9, 774-784.

(77) Akimov, S. A.; Volynsky, P. E.; Galimzyanov, T. R.; Kuzmin, P. I.; Pavlov, K. V.; Batishchev, O. V. Pore formation in lipid membrane I: Continuous reversible trajectory from intact bilayer through hydrophobic defect to transversal pore. Sci. Rep. 2017, 7, 12152.

(78) Kawamoto, S.; Shinoda, W. Free energy analysis along the stalk mechanism of membrane fusion. Soft Matter 2014, 10, 3048-3054.

(79) Shinoda, W.; DeVane, R.; Klein, M. L. Zwitterionic Lipid Assemblies: Molecular Dynamics Studies of Monolayers, Bilayers, and Vesicles Using a New Coarse Grain Force Field. J. Phys. Chem. B 2010, 114, 6836-6849.

(80) Awasthi, N.; Kopec, W.; Wilkosz, N.; Jamróz, D.; Hub, J. S.; Zatorska, M.; Petka, R.; Nowakowska, M.; Kepczynski, M. Molecular Mechanism of Polycation-Induced Pore Formation in Biomembranes. ACS Biomater. Sci. Eng. 2019, 5, 780-794.

(81) Neale, C.; Pomes, R. Sampling errors in free energy simulations of small molecules in lipid bilayers. Biosimulations of lipid membranes coupled to experiments 1858, 2016, 2539-2548. 
(82) Pearlman, D. A.; Kollman, P. A. The lag between the Hamiltonian and the system configuration in free energy perturbation calculations. J. Chem. Phys. 1989, 91, 7831-7839.

(83) López, C. A.; Sovova, Z.; van Eerden, F. J.; de Vries, A. H.; Marrink, S. J. Martini Force Field Parameters for Glycolipids. J. Chem. Theory Comput. 2013, 9, 1694-1708.

(84) Ingólffsson, H. I.; Melo, M. N.; van Eerden, F. J.; Arnarez, C.; Lopez, C. A.; Wassenaar, T. A.; Periole, X.; de Vries, A. H.; Tieleman, D. P.; Marrink, S. J. Lipid Organization of the Plasma Membrane. J. Am. Chem. Soc. 2014, 136, 14554-14559.

(85) Marrink, S. J.; de Vries, A. H.; Mark, A. E. Coarse Grained Model for Semiquantitative Lipid Simulations. J. Phys. Chem. B 2004, 108, 750-760.

(86) Marrink, S. J.; Risselada, H. J.; Yefimov, S.; Tieleman, D. P.; de Vries, A. H. The MARTINI Force Field: Coarse Grained Model for Biomolecular Simulations. J. Phys. Chem. B 2007, 111, 7812-7824.

(87) Pérez-Lara, A.; Thapa, A.; Nyenhuis, S. B.; Nyenhuis, D. A.; Halder, P.; Tietzel, M.; Tittmann, K.; Cafiso, D. S.; Jahn, R. PtdInsP ${ }_{2}$ and PtdSer cooperate to trap synaptotagmin-1 to the plasma membrane in the presence of calcium. eLife 2016, 5, No. e15886.

(88) Calejo, A. I.; Jorgacevski, J.; Kucka, M.; Kreft, M.; Goncalves, P. P.; Stojilkovic, S. S.; Zorec, R. cAMP-mediated stabilization of fusion pores in cultured rat pituitary lactotrophs. J. Neurosci. 2013, 33, 8068-8078.

(89) Finkenstaedt-Quinn, S. A.; Gruba, S. M.; Haynes, C. L. Variations in Fusion Pore Formation in Cholesterol-Treated Platelets. Biophys. J. 2016, 110, 922-929.

(90) Mackenzie, K. D.; Duffield, M. D.; Peiris, H.; Phillips, L.; Zanin, M. P.; Teo, E. H.; Zhou, X.-F.; Keating, D. J. Huntingtin-associated protein 1 regulates exocytosis, vesicle docking, readily releasable pool size and fusion pore stability in mouse chromaffin cells. J. Physiol 2014, 592, 1505-1518.

(91) Zanin, M. P.; Phillips, L.; Mackenzie, K. D.; Keating, D. J. Aging differentially affects multiple aspects of vesicle fusion kinetics. PLoS One 2011, 6, e27820-e27820.

(92) Guček, A.; Jorgačevski, J.; Singh, P.; Geisler, C.; Lisjak, M.; Vardjan, N.; Kreft, M.; Egner, A.; Zorec, R. Dominant negative SNARE peptides stabilize the fusion pore in a narrow, releaseunproductive state. Cell. Mol. Life Sci. 2016, 73, 3719-3731.

(93) Jo, S.; Kim, T.; Iyer, V. G.; Im, W. CHARMM-GUI: A webbased graphical user interface for CHARMM. J. Comput. Chem. 2008, $29,1859-1865$

(94) Van Der Spoel, D.; Lindahl, E.; Hess, B.; Groenhof, G.; Mark, A. E.; Berendsen, H. J. C. GROMACS: Fast, flexible, and free. J. Comput. Chem. 2005, 26, 1701-1718.

(95) Pronk, S.; Páll, S.; Schulz, R.; Larsson, P.; Bjelkmar, P.; Apostolov, R.; Shirts, M. R.; Smith, J. C.; Kasson, P. M.; van der Spoel, D.; et al. GROMACS 4.5: a high-throughput and highly parallel open source molecular simulation toolkit. Bioinformatics 2013, 29, 845-854.

(96) Abraham, M. J.; Murtola, T.; Schulz, R.; Páll, S.; Smith, J. C.; Hess, B.; Lindahl, E. GROMACS: High performance molecular simulations through multi-level parallelism from laptops to supercomputers. SoftwareX 2015, 1-2, 19-25.

(97) Herzog, F. A.; Braun, L.; Schoen, I.; Vogel, V. Improved Side Chain Dynamics in MARTINI Simulations of Protein-Lipid Interfaces. J. Chem. Theory Comput. 2016, 12, 2446-2458.

(98) van Eerden, F. J.; van den Berg, T.; Frederix, P. W. J. M.; de Jong, D. H.; Periole, X.; Marrink, S. J. Molecular Dynamics of Photosystem II Embedded in the Thylakoid Membrane. J. Phys. Chem. B 2016, 3237-3249.

(99) Damre, M.; Marchetto, A.; Giorgetti, A. MERMAID: dedicated web server to prepare and run coarse-grained membrane protein dynamics. Nucleic Acids Res. 2019, 47, W456-W461.

(100) Hall, B. A.; Chetwynd, A. P.; Sansom, M. S. P. Exploring peptide-membrane interactions with coarse-grained $\mathrm{MD}$ simulations. Biophys. J. 2011, 100, 1940-1948.
(101) Javanainen, M.; Martinez-Seara, H.; Vattulainen, I. Excessive aggregation of membrane proteins in the Martini model. PLoS One 2017, 12, No. e0187936.

(102) de Jong, D. H.; Singh, G.; Bennett, W. F. D.; Arnarez, C.; Wassenaar, T. A.; Schäfer, L. V.; Periole, X.; Tieleman, D. P.; Marrink, $S$. J. Improved parameters for the martini coarse-grained protein force field. J. Chem. Theory Comput. 2012, 9, 687-697.

(103) Marrink, S. J.; Mark, A. E. The Mechanism of Vesicle Fusion as Revealed by Molecular Dynamics Simulations. J. Am. Chem. Soc. 2003, 125, 11144-11145.

(104) Marrink, S. J.; de Vries, A. H.; Tieleman, D. P. Lipids on the move: Simulations of membrane pores, domains, stalks and curves. Biochim. Biophys. Acta, Biomembr. 2009, 1788, 149-168.

(105) Baaden, M.; Marrink, S. J. Coarse-grain modelling of proteinprotein interactions. Curr. Opin. Struct. Biol. 2013, 23, 878-886.

(106) Uusitalo, J. J.; Ingólfsson, H. I.; Akhshi, P.; Tieleman, D. P.; Marrink, S. J. Martini coarse-grained force field: extension to DNA. J. Chem. Theory Comput. 2015, 11, 3932-3945.

(107) Masone, D.; Bustos, D. M. Transmembrane domain dimerization induces cholesterol rafts in curved lipid bilayers. Phys. Chem. Chem. Phys. 2019, 21, 268-274.

(108) Yesylevskyy, S. O.; Schäfer, L. V.; Sengupta, D.; Marrink, S. J. Polarizable Water Model for the Coarse-Grained MARTINI Force Field. PLoS Comput. Biol. 2010, 6, 1-17.

(109) Alessandri, R.; Souza, P. C. T.; Thallmair, S.; Melo, M. N.; de Vries, A. H.; Marrink, S. J. Pitfalls of the Martini Model. J. Chem. Theory Comput. 2019, 15, 5448-5460.

(110) Caparotta, M.; Bustos, D. M.; Masone, D. Order-disorder skewness in alpha-synuclein: a key mechanism to recognize membrane curvature. Phys. Chem. Chem. Phys. 2020, 22, 5255-5263.

(111) Masone, D.; Uhart, M.; Bustos, D. M. Bending Lipid Bilayers: A Closed-Form Collective Variable for Effective Free-Energy Landscapes in Quantitative Biology. J. Chem. Theory Comput. 2018, $14,2240-2245$

(112) Jo, S.; Lim, J. B.; Klauda, J. B.; Im, W. CHARMM-GUI Membrane Builder for Mixed Bilayers and Its Application to Yeast Membranes. Biophys. J. 2009, 97, 50-58.

(113) Rui, H.; Lee, K. I.; Pastor, R. W.; Im, W. Molecular Dynamics Studies of Ion Permeation in VDAC. Biophys. J. 2011, 100, 602-610.

(114) Jo, S.; Kim, T.; Im, W. Automated Builder and Database of Protein/Membrane Complexes for Molecular Dynamics Simulations. PLoS One 2007, 2, e880.

(115) Bussi, G.; Donadio, D.; Parrinello, M. Canonical sampling through velocity rescaling. J. Chem. Phys. 2007, 126, No. 014101.

(116) Seelig, A.; Seelig, J. Dynamic structure of fatty acyl chains in a phospholipid bilayer measured by deuterium magnetic resonance. Biochemistry 1974, 13, 4839-4845.

(117) Porasso, R. D.; Ale, N. M.; Ciocco Aloia, F.; Masone, D.; Del Pópolo, M. G.; Altabef, A. B.; Gomez-Zavaglia, A.; Diaz, S. B.; Vila, J. A. Interaction of glycine, lysine, proline and histidine with dipalmitoylphosphatidylcholine lipid bilayers: a theoretical and experimental study. RSC Adv. 2015, 5, 43537-43546.

(118) Berendsen, H. J. C.; Postma, J. P. M.; van Gunsteren, W. F.; DiNola, A.; Haak, J. R. Molecular dynamics with coupling to an external bath. J. Chem. Phys. 1984, 81, 3684.

(119) Humphrey, W.; Dalke, A.; Schulten, K. VMD - Visual Molecular Dynamics. Journal of Molecular Graphics 1996, 14, 33-38. (120) SchrodingerLLC; Schrodinger: LLC, New York, NY, 2014.

(121) Kumar, S.; Rosenberg, J. M.; Bouzida, D.; Swendsen, R. H.; Kollman, P. A. The weighted histogram analysis method for freeenergy calculations on biomolecules I. The method. J. Comput. Chem. 1992, 13, 1011-1021. 